\title{
Intelligence and Variability in a Simple Timing Task Share Neural Substrates in the Prefrontal White Matter
}

\author{
Fredrik Ullén, ${ }^{1}$ Lea Forsman, ${ }^{1}$ Örjan Blom, ${ }^{1}$ Anke Karabanov, ${ }^{1}$ and Guy Madison ${ }^{2}$ \\ ${ }^{1}$ Department of Woman and Child Health, Karolinska Institutet, SE-171 77 Stockholm, Sweden, and ²Department of Psychology, Umeå University, SE-901 \\ 87 Umeå, Sweden
}

\begin{abstract}
General intelligence is correlated with the mean and variability of reaction time in elementary cognitive tasks, as well as with performance on temporal judgment and discrimination tasks. This suggests a link between the temporal accuracy of neural activity and intelligence. However, it has remained unclear whether this link reflects top-down mechanisms such as attentional control and cognitive strategies or basic neural properties that influence both abilities. Here, we investigated whether millisecond variability in a simple, automatic timing task, isochronous tapping, correlates with intellectual performance and, using voxel-based morphometry, whether these two tasks share neuroanatomical substrates. Stability of tapping and intelligence were correlated and related to regional volume in overlapping right prefrontal white matter regions. These results suggest a bottom-up explanation of the link between temporal stability and intellectual performance, in which more extensive prefrontal connectivity underlies individual differences in both variables.
\end{abstract}

Key words: cognition; morphometry; motor control; prefrontal; temporal; white matter

\section{Introduction}

Recent years have witnessed an increased interest in the neurobiological underpinnings of psychometric general intelligence $(g)$ (Deary, 2000; Jung and Haier, 2007). Neuroimaging studies have thus shown that intelligence is related to both structural and functional brain properties. The most well replicated anatomical finding is a positive correlation $(r=0.3-0.4)$ between intelligence and measures of total brain volume (McDaniel, 2005; Rushton and Ankney, 2007). Several studies have also investigated the relationship between regional brain anatomy and intelligence (Jung and Haier, 2007), demonstrating positive correlations between intelligence and regional brain volume in widespread areas of the gray and the white matter but particularly in prefrontal and temporoparietal association cortices (Reiss et al., 1996; Frangou et al., 2004; Haier et al., 2004, 2005; Colom et al., 2006; Shaw et al., 2006).

These results are in line with findings from functional neuroimaging studies, in which brain activity during performance of highly g-loaded tasks was measured. Solving items from intelligence tests (Haier et al., 1988; Prabhakaran et al., 1997; Duncan et al., 2000) or performing other highly $g$-loaded reasoning tasks (Goel et al., 1997; Goel and Dolan, 2001; Kroger et al., 2002; Geake and Hansen, 2005) is accompanied by activity in widespread regions, but involvement of prefrontal and parietal cortices is one of the most consistent findings across studies (Jung and

Received Dec. 28, 2007; revised March 11, 2008; accepted March 12, 2008.

This work was funded by the Swedish Research Foundation and the Söderberg Foundation. We are grateful to Hans Forssberg, Mark Hallett, and Sara L. Bengtsson for comments on a previous version of this manuscript and to Jesper Andersson for discussions of statistical issues.

Correspondence should be addressed to Fredrik Ullén, Neuropediatric Research Unit Q2:07, Department of Woman and Child Health, Karolinska Institutet, SE-171 76 Stockholm, Sweden. E-mail: fredrik.ullen@ki.se. DOI:10.1523/JNEUROSCI.0825-08.2008

Copyright $\odot 2008$ Society for Neuroscience $\quad 0270-6474 / 08 / 284238-06 \$ 15.00 / 0$
Haier, 2007). A comprehensive review and synthesis of the neuroimaging literature on intelligence can be found in the paper by Jung and Haier (2007), in which information processing in frontoparietal networks is proposed to be a central mechanism of intellectual functioning.

A different approach to the study of the basis of intelligence has its origins in the psychometric tradition: chronometric studies of performance in elementary cognitive tasks. A consistent finding in such studies is that intelligence correlates negatively with the mean and variability of reaction time (RT) in a wide range of elementary cognitive tasks (Deary, 2001; Jensen, 2006). In several studies, RT variability has shown a somewhat larger negative correlation with intelligence than mean RT (Jensen, 1992; Baumeister, 1998; Deary, 2001). Recent studies including RT tasks, as well as temporal discrimination and judgment tasks, have reported that the latter tasks correlate better with intelligence than RT and that the portion of overall variability in intelligence explained by the RT tasks almost entirely represents variance also explained by the temporal tasks (Helmbold et al., 2007; Rammsayer and Brandler, 2007). Neural factors influencing accuracy of timing may thus be fundamental to intelligence. However, previously used temporal tasks included explicit manipulation of time intervals in working memory (Helmbold et al., 2007; Rammsayer and Brandler, 2007). The link between intelligence and timing could thus plausibly be explained by top-down mechanisms such as attentional control and cognitive strategies as well as by bottom-up mechanisms, e.g., in terms of basic neural properties that influence both abilities (Deary, 2001). An important question is therefore whether temporal accuracy in simple, automatic timing tasks that load minimally on working memory and executive control are correlated with intellectual performance and, if so, whether these two behaviors share neural correlates.

We investigated this question by collecting neuroanatomical, 
psychometric, and chronometric measurements from the same group of participants. Studying structural rather than functional correlates of intelligence was deemed advantageous in the present context: structural correlates are task independent and can reveal anatomical features related to intelligence in the whole brain, whereas patterns of brain activity also depend on which neural circuits are recruited by the task under study (Jung and Haier, 2007). A high-resolution T1-weighted anatomical magnetic resonance image of the whole brain was collected from each participant and analyzed using voxel-based morphometry (Good et al., 2001). Intelligence was assessed with the Raven SPM Plus. The timing task was isochronous interval production (tapping). This is a simple timing task that does not include processing of durations in working memory or response selection and information processing of the type typically required in the elementary cognitive tasks (Deary, 2001). Indeed, a number of studies suggest that interval-to-interval variability in isochronous tapping is controlled by automatic processes. Temporal adaptations to distractors (Repp, 2006) or perturbations of pacing stimuli (Madison and Merker, 2004) occur unintentionally, involuntarily and without perceptual awareness. We used the coefficient of variation $(\mathrm{CV})$ of differences between successive interval durations as a measure of temporal variability, which reflects local interval-tointerval variability with minimal influence of drift and other higher-order dependencies (Madison, 2001). The neuroanatomical correlates of intelligence and tapping variability, and their overlap, were investigated with regression analyses.

\section{Materials and Methods}

Participants. Thirty-four right-handed (Oldfield, 1971) males with no history of neurological disease participated in the study (age, 19-49 years; mean age, $33.0 \pm 7.9$ years). The participants were recruited from the Stockholm area with a newspaper advertisement. Imaging data from two subjects was excluded because of head movement artifacts. The tapping data from two participants could not be recorded because of a technical failure. In other words, $n=30$ participants were included in analyses of tapping variability and brain anatomy, whereas in all other analyses, $n=32$ participants were used. The experimental procedures were undertaken with the understanding and written consent of each participant, conformed to The Code of Ethics of the World Medical Association (Declaration of Helsinki), and were ethically approved by the Karolinska Hospital Ethical Committee (Dnr 2005/320-32).

Psychometric testing. Intelligence was measured with the SPM Plus version (60 items) of the Raven's Progressive Matrices (Styles et al., 1998), a widely used test that mainly reflects psychometric general intelligence $(g)$ (Gustafsson, 1984). The test was administered individually without time limit. Obtained scores ranged between 34 and 58, with a mean score of $46.1 \pm 7.4$.

Isochronous tapping task. The experiment was controlled by customdesigned software running on a personal computer with a real-time operating system. An Alesis D4 drum module connected via MIDI to the personal computer produced the sounds and collected the responses. Stimuli consisted of 20 sampled cowbell sounds presented in isochronous sequence through Peltor HTB7A sound-attenuated headphones at $\sim 78 \mathrm{dBA}$ sound pressure level, having a suprathreshold duration of $\sim 80$ ms. Sounds 19 and 20 were attenuated to 72 and $66 \mathrm{dBA}$, respectively, to reduce the startle reaction when stimuli cease. Responses were given by beating a drumstick against a drum pad with a piezoelectric element.

Each participant was tested individually, sitting upright on a chair with the feet placed evenly on the floor. In each trial, the participant synchronized right-hand tapping movements with 20 auditory metronome clicks and then continued to tap another 45 times without interruption after the metronome had stopped. This procedure was repeated for 14 trials, using seven different metronome interonset intervals $(215,300,375,469$, 586, 733, and $916 \mathrm{~ms}$ ), each replicated twice and presented in random order. The first block consisted of seven trials, one for each interonset interval, and the second of 14 trials, two for each interonset interval. The first block was for practice, and those data were ignored. Trials within each block were given in a different random order for each participant. The 39 temporal intervals between the last 40 taps in each continuation phase were analyzed. First, a measure of interval-to-interval variability, "local," was calculated for each trial as follows:

$$
\text { local }=\left[\frac{\sum_{1}^{N-2}\left(x_{i+2}-x_{i}\right)^{2}}{2(N-2)}\right]^{0.5},
$$

where $x_{i}$ is the duration of the temporal interval between tap $i$ and tap $i+$ 1 , and $N$ is the number of intervals in a trial (i.e., 39). The measure local is comparable with the $\mathrm{SD}$, the essential difference being that it is based on local differences between data points instead of differences between data and their global mean. It is therefore a measure of variability that is minimally influenced by gradual changes of tapping frequency (drift) and other higher-order dependencies. Because tapping sequences are known to exhibit lag 1 negative dependency (Vorberg and Wing, 1996), lag 2 instead of lag 1 differences are used in the calculation of local, which is why $N-2$ must be used instead of $N-1$. If we consider random data, sequential differences are on average twice as large as differences from the mean, which is why the denominator is twice as large as for the computation of SD. Because variability increases with the interval, local was divided by the mean interval for each trial to obtain the CV. The metric on which correlations were computed was the mean $\mathrm{CV}$ across all trials $(n=14)$ within each subject.

Magnetic resonance imaging. Imaging was performed using a $1.5 \mathrm{~T}$ scanner (Signa Horizon Echospeed; GE Medical Systems, Madison, WI) with a standard eight-channel head coil. A three-dimensional, highresolution T1-weighted anatomical image volume was acquired from each subject, with the following parameters: acquisition matrix, $256 \times$ $256 \mathrm{~mm}$; field of view, $25 \mathrm{~cm}$; repetition time, $24 \mathrm{~ms}$; echo time, $6 \mathrm{~ms}$; flip angle, $30^{\circ}$; number of slices, 150 ; slice thickness, $1 \mathrm{~mm}$; and voxel size, $1 \times 1 \times 1 \mathrm{~mm}^{3}$.

The magnetic resonance images were processed for voxel-based morphometry using the VBM2 toolbox (Cuadra et al., 2005) (http://dbm. neuro.uni-jena.de/vbm) within the SPM2 software package (Wellcome Department of Cognitive Neurology, London, UK). The preprocessing of the images was performed as described previously, using studyspecific prior probability maps (Good et al., 2001). All images were thus spatially normalized to standardized anatomical space and segmented into separate images of gray matter (GM), white matter, and CSF. The segmentation procedure was optimized by including the hidden Markov random field (HMRF)-based algorithm implemented in VBM2. This procedure removes isolated voxels of one tissue class, which are unlikely to be true members of this tissue type, judging from the tissue class of neighboring voxels. A HMRF weighting of 0.3 was used. Images were modulated, i.e., voxel values were multiplied with Jacobian determinants from the normalization procedure, so that they reflected regional differences in absolute amount (volume) of gray and white matter (Good et al., 2001). The resulting gray and white matter images were smoothed with a Gaussian kernel of $12 \mathrm{~mm}$ full-width half-maximum before analysis.

Modulated gray and white matter images were regressed, using the general linear model, on Raven scores or on CV scores from the isochronous tapping task. The covariates were corrected to have a 0 mean. In all models, voxels with a value of $<0.2$ were excluded to avoid edge effects around the border between gray and white matter (Mühlau et al., 2006). Statistical significance was determined using a voxel height threshold of $p<0.05$, corrected for multiple comparisons using false discovery rate (Genovese et al., 2002). To localize white matter regions with overlapping correlations with both Raven scores and CV scores, white matter density was first regressed on CV ( $p<0.05$, corrected). These clusters were used as an inclusive explicit mask in a second regression model between white matter volume (WMV) and Raven scores. All regressions were also performed with age included as a nuisance covariate, to control for age effects.

For the clusters with overlapping correlations with Raven and CV 
scores, a commonality analysis (Seibold and McPhee, 1979) was performed to determine the proportion of total variance in Raven scores associated with WMV and CV scores uniquely, as well as with common effects of these variables. The commonality $(c)$ was calculated as: $c=$ $r_{\text {Raven.WMV }}^{2}+r_{\text {Raven.CV }}^{2}-R_{\text {Raven.WMV,CV }}^{2}$ where $r^{2}{ }_{\text {Raven.WMV }}$ is the squared correlation coefficient between Raven scores and WMV, $r_{\text {Raven.CV }}^{2}$ is the squared correlation coefficient between Raven scores and CV, and $R_{\text {Raven.WMV,CV }}^{2}$ is the squared multiple correlation coefficient with Raven scores as dependent variable and WMV and CV as independent variables. Unique contributions of WMV $\left(\mathrm{u}_{\mathrm{WMV}}\right)$ and $\mathrm{CV}\left(\mathrm{u}_{\mathrm{CV}}\right)$ were calculated as follows: $\mathrm{u}_{\mathrm{WMV}}$ $=R_{\text {Raven.WMV,CV }}^{2}-r_{\text {Raven.CV }}^{2}$, and $\mathrm{u}_{\mathrm{CV}}=$ $R_{\text {Raven.WMV,CV }}^{2}-r_{\text {Raven.WMV. }}^{2}$

\section{Results}

$\mathrm{CV}$ and intelligence were negatively correlated $(r=-0.39, p=0.03$, two-tailed test; $r=-0.48, p=0.006$ when controlling for age). Correlations with brain anatomy were first investigated with exploratory analyses in the whole GM and WM volumes. Positive correlations between intelligence and GM volume were widespread but most extensive in frontal and parietotemporal association areas, in particular right prefrontal cortex (Fig. 1, Table 1). When controlling for age, trends at $p=0.07-0.08$ were found in the same clusters (Table 1 ). Trends for positive correlations between intelligence and WM volume did not reach significance $(p=0.12)$ in a whole-brain search. No negative correlations were found between intelligence and GM or WM volume in any regions. CV correlated negatively with WM volume in frontal, temporal, and parietal fiber tracts (Table 2). These correlations were still significant when controlling for age, with the exception of a few peaks in which strong trends at $p=0.06-0.07$ were found (Table 2). No negative correlations were found between $\mathrm{CV}$ and regional GM volume, nor were any positive correlations found between CV and GM or WM volume in any regions.

Second, we investigated the overlap between intelligence and $\mathrm{CV}$ correlates. When reducing the search volume to those WM voxels that correlated negatively with $\mathrm{CV}$, significant positive correlations with Raven scores were found in right dorsal and ventral prefrontal fiber tracts and in a small cluster in left ventral prefrontal cortex (Fig. 2, Table 3). All clusters remained significant when controlling for age. Table 3 also shows, for each cluster, commonality analyses of multiple regressions of with intelligence as dependent variable and CV and regional white matter volume as independent variables. Cluster volume and CV commonly predicted $15 \%$ of the intelligence variance, whereas cluster volume uniquely predicted an additional $7-15 \%$ of intelligence. There were no unique contributions of $\mathrm{CV}$ to intelligence. No significant correlations with $\mathrm{CV}$ were found in the GM voxels that correlated with intelligence.

\section{Discussion}

The correlations between Raven scores and regional gray matter volume found here are consistent with other recent reports of positive correlations between psychometric intelligence and regional brain volume in widespread cortical areas (Reiss et al., 1996; Frangou et al., 2004; Haier et al., 2004, 2005; Colom et al.,
2006; Shaw et al., 2006). The overall pattern of correlations, with large clusters in right prefrontal and temporoparietal association areas, replicates remarkably well the pattern found in another study using a similar sample of adult male subjects (Haier et al., 2005). No previous studies have investigated neuroanatomical correlates of tapping variability. However, a negative correlation between intraindividual variability in reaction time and total WMV has been reported recently (Walhovd and Fjell, 2007), supporting that the amount of white matter is related to trial-totrial variability in timed tasks.

The present study makes two important new advances. First, we demonstrate that intelligence is related to millisecond accuracy in isochronous tapping, a simple timing task that does not involve response selection or information processing of the type typically required in the elementary cognitive tasks (Deary, 2001) and in which interval-to-interval variability is primarily controlled by automatic processes (see Introduction). Second, we show that tapping variability and intelligence share neural substrates in the prefrontal white substance. Commonality analyses showed that the portion of intelligence variability explained by tapping variability was entirely shared with regional white matter volume, whereas the latter variable also had unique contributions to intelligence variability. A larger amount of right prefrontal white matter may thus be the common neurobiological factor underlying correlated individual differences in temporal accuracy and intellectual performance.

This notion is in line with a general parietofrontal integration model of intelligence (Jung and Haier, 2007) but specifically suggests a bottom-up explanation for the link between chronometric and psychometric tasks: more extensive right prefrontal connectivity causes better performance in both types of behavior. Indeed, it has been proposed recently that the right prefrontal cortex contains neural circuits that are critical for both timing tasks and working memory, on the basis of convergent evidence from 
Table 1. Gray matter areas with a positive correlation between intelligence and regional volume

\begin{tabular}{|c|c|c|c|c|c|c|}
\hline Brain region & Side & $t$ value & $x$ & $y$ & $z$ & Size $\left(\mathrm{mm}^{3}\right)$ \\
\hline \multicolumn{7}{|l|}{ Frontal lobe } \\
\hline \multirow[t]{3}{*}{ Inf frontal g } & \multirow[t]{3}{*}{$\mathrm{R}$} & 5.31 & 29 & 65 & -2 & \multirow[t]{3}{*}{$18462^{a}$} \\
\hline & & 5.00 & 51 & 41 & -12 & \\
\hline & & 5.25 & 58 & 25 & 1 & \\
\hline Middle frontal g & L & $3.11^{b}$ & -31 & 61 & 5 & 39 \\
\hline Sup frontal g & L & 3.10 & -18 & 60 & 6 & 21 \\
\hline \multirow[t]{2}{*}{ Sup frontal g } & \multirow[t]{2}{*}{$\mathrm{R}$} & 4.00 & 7 & 59 & 4 & \multirow[t]{2}{*}{$6287^{c}$} \\
\hline & & 3.79 & 9 & 58 & 12 & \\
\hline Cingulate g & $\mathrm{R}$ & 4.03 & 9 & 53 & -5 & \multirow{4}{*}{653} \\
\hline \multirow[t]{3}{*}{ Inf frontal g } & \multirow[t]{3}{*}{ L } & 4.24 & -48 & 40 & -16 & \\
\hline & & 3.25 & -51 & 33 & -11 & \\
\hline & & $3.11^{b}$ & -55 & 30 & -4 & \\
\hline Inf frontal g & L & 3.19 & -58 & 22 & 11 & 15 \\
\hline Middle frontal g & $\mathrm{R}$ & 3.18 & 45 & 18 & 48 & \multirow[t]{3}{*}{280} \\
\hline \multirow[t]{2}{*}{ Precentral g } & \multirow[t]{2}{*}{$\mathrm{R}$} & 3.26 & 49 & -1 & 50 & \\
\hline & & 3.47 & 44 & -7 & 46 & \\
\hline \multirow[t]{2}{*}{ Inf frontal g } & \multirow[t]{2}{*}{ L } & 3.58 & -43 & 15 & 8 & \multirow[t]{2}{*}{2057} \\
\hline & & 3.25 & -51 & 11 & 13 & \\
\hline 3.74 & -56 & 9 & 4 & & & \multirow{4}{*}{3131} \\
\hline \multirow{3}{*}{ Cingulate g } & \multirow[t]{3}{*}{ L } & 4.57 & -9 & 9 & 31 & \\
\hline & & 4.25 & -9 & -29 & 32 & \\
\hline & & 3.57 & -7 & -19 & 32 & \\
\hline Sup frontal g & Mid & 4.31 & -3 & -10 & 72 & 1332 \\
\hline Precentral g & 3.09 & 1 & -23 & 69 & & \\
\hline \multirow[t]{2}{*}{ Cingulate g } & $\mathrm{R}$ & 3.46 & 10 & -15 & 35 & \multirow[t]{2}{*}{274} \\
\hline & & 3.02 & 12 & -22 & 41 & \\
\hline \multicolumn{7}{|l|}{ Parietal and temporal lobes } \\
\hline Postcentral g & $\mathrm{L}$ & 3.33 & -64 & -9 & 22 & 54 \\
\hline Supramarginal g & $\mathrm{R}$ & 5.19 & 50 & -38 & 26 & $26549^{d}$ \\
\hline Middle temporal g & 4.41 & 50 & -46 & 1 & & \\
\hline Supramarginal g & 4.44 & 51 & -55 & 42 & & \\
\hline \multirow[t]{2}{*}{ Sup temporal g } & \multirow[t]{2}{*}{$\mathrm{L}$} & 3.02 & -47 & -38 & 11 & 1114 \\
\hline & & 3.84 & -56 & -41 & 16 & \\
\hline Inf temporal g & $\mathrm{L}$ & 3.40 & -63 & -58 & -8 & 4839 \\
\hline Middle temporal g & $\mathrm{L}$ & 4.31 & -61 & -65 & -1 & \\
\hline & & 4.81 & -53 & -68 & 4 & \\
\hline Angular g & $\mathrm{R}$ & 3.21 & 44 & -58 & 24 & 39 \\
\hline Angularg & L & 3.08 & -56 & -62 & 35 & 27 \\
\hline Angular g & L & 4.18 & -44 & -72 & 26 & $1069^{e}$ \\
\hline Subcortical & & & & & & \\
\hline Hypothalamus & Mid & 3.10 & -1 & 7 & -16 & 312 \\
\hline & & 3.56 & -3 & -3 & -18 & \\
\hline Cerebellum, lobule VI & L & $3.06^{b}$ & -28 & -51 & -27 & 38 \\
\hline
\end{tabular}

Significantly correlated $(p<0.05)$ regions with an extent of more than five voxels are reported, in rostrocaudal order within each lobe. The coordinates in Montreal Neurological Institute (MNI) space and $t$ values of the three most significant peak voxels of each cluster are given. When controlling for age, trends at $p=0.08$ were found for all peaks, except for three peaks that were significant at $p=0.09$ (see footnote at the respective $t$ value). g, Gyrus; Inf, inferior; Sup, superior; L, left; R, right.

${ }^{a}$ The cluster extended rostrocaudally from $y=6$ in the inferior frontal gyrus to the frontal pole; in its rostral part, it extended dorsally into the right middle and superior frontal gyri and ventromedially into orbitofrontal cortex.

${ }^{b}$ The peak had a trend for significance at $p=0.09$ when controlling for age.

The cluster extended caudally in the right cingulate gyrus to $y=16$.

${ }^{d}$ The cluster extended dorsoventrally from the postcentral sulcus to the inferior temporal and fusiform gyri, in posterior parietotemporal cortex.

${ }^{e}$ The cluster extended caudally into the middle occipital gyrus.

neuroimaging studies, pharmacological experiments, and animal electrophysiology (Lewis and Miall, 2006). Working memory capacity is closely related to psychometric general intelligence (Kyllonen and Christal, 1990). It may seem paradoxical that repetitive motor timing tasks, such as the tapping task used here, involve prefrontal mechanisms less than perceptual timing tasks that do not involve repetitive production of rhythms (Lewis and Miall, 2003). However, individual difference studies have demonstrated that tapping variability is substantially correlated with
Table 2. White matter areas with a negative correlation between tapping variability (CV) and regional volume

\begin{tabular}{|c|c|c|c|c|c|c|}
\hline Brain region (nearest gray matter) & Side & $t$ value & $x$ & $y$ & $z$ & Size $\left(\mathrm{mm}^{3}\right)$ \\
\hline \multicolumn{7}{|l|}{ Frontal lobe } \\
\hline \multirow[t]{2}{*}{ Sup frontal g } & \multirow[t]{2}{*}{ L } & 4.76 & -15 & 55 & 22 & \multirow[t]{2}{*}{2122} \\
\hline & & 3.80 & -13 & 57 & 13 & \\
\hline Orbitofrontal g & L & 3.48 & -9 & 55 & -8 & \\
\hline Inf frontal g & $\mathrm{R}$ & 4.15 & 36 & 52 & -1 & $9408^{a}$ \\
\hline Orbitofrontal g & $\mathrm{R}$ & 4.04 & 9 & 54 & -13 & \\
\hline Inf frontal g & $\mathrm{R}$ & 3.98 & 26 & 39 & -10 & \\
\hline Sup frontal g & $\mathrm{R}$ & $3.25^{b}$ & 9 & 53 & 17 & 85 \\
\hline \multirow[t]{2}{*}{ Orbitofrontal g } & \multirow[t]{2}{*}{ L } & 3.85 & -23 & 36 & -15 & \multirow[t]{2}{*}{1189} \\
\hline & & 3.40 & -27 & 42 & -9 & \\
\hline Sup frontal g & L & $3.42^{b}$ & -12 & 20 & 58 & 27 \\
\hline Middle frontal g & $\mathrm{R}$ & $3.27^{c}$ & 32 & 8 & 51 & 84 \\
\hline \multirow[t]{3}{*}{ Precentral g } & \multirow[t]{3}{*}{ L } & 4.34 & -44 & -1 & 16 & \multirow[t]{3}{*}{$9560^{d}$} \\
\hline & & 4.30 & -47 & -16 & 20 & \\
\hline & & 4.28 & -35 & -1 & 25 & \\
\hline Precentral g & $\mathrm{R}$ & 4.42 & 47 & -6 & 19 & 1288 \\
\hline Internal capsule, posterior limb & $\mathrm{L}$ & 3.76 & -18 & -8 & -3 & 1318 \\
\hline Sup frontal g & $\mathrm{R}$ & 3.68 & 7 & -16 & 61 & 131 \\
\hline Precentral g & R & $3.20^{b}$ & 40 & -23 & 65 & 20 \\
\hline \multicolumn{7}{|l|}{ Parietal and temporal lobes } \\
\hline \multirow[t]{3}{*}{ Inf temporal g } & \multirow[t]{3}{*}{$\mathrm{R}$} & 3.83 & 40 & -19 & -24 & \multirow[t]{3}{*}{1905} \\
\hline & & 3.68 & 46 & -12 & -26 & \\
\hline & & 3.18 & 39 & -6 & -31 & \\
\hline \multirow[t]{2}{*}{ Inf temporal g } & \multirow[t]{2}{*}{ L } & 4.80 & -41 & -14 & -24 & \multirow[t]{2}{*}{3423} \\
\hline & & 4.57 & -41 & -7 & -29 & \\
\hline \multirow[t]{2}{*}{ Supramarginal g } & $\mathrm{R}$ & 4.94 & 54 & -25 & 37 & 1310 \\
\hline & L & 3.26 & -48 & -41 & 36 & 37 \\
\hline \multirow[t]{2}{*}{ Precuneus } & \multirow[t]{2}{*}{ R } & 4.45 & 12 & -54 & 31 & \multirow[t]{2}{*}{1260} \\
\hline & & 3.22 & 18 & -50 & 15 & \\
\hline \multirow[t]{2}{*}{ Angular g } & L & 4.08 & -39 & -66 & 31 & \multirow[t]{2}{*}{670} \\
\hline & & $3.29^{b}$ & -29 & -71 & 20 & \\
\hline
\end{tabular}

Significantly correlated $(p<0.05)$ regions with an extent of more than five voxels are reported, in rostrocaudal order within each lobe. The coordinates in MNI space and $t$ values of the three most significant peak voxels of each cluster are given. All peaks remained significant at $p=0.05$ when controlling for age, with a few exceptions in which strong trends for significance were found (see footnotes at the corresponding $t$ values). g, Gyrus; Inf, inferior; Sup, superior; L, left; $R$, right.

${ }^{a}$ The cluster extended dorsally into the middle and superior frontal gyri.

${ }^{b}$ The peak had a trend for significance at $p=0.06$ when controlling for age.

'The peak had a trend for significance at $p=0.07$ when controlling for age.

${ }^{d}$ The cluster extended ventrally into the inferior frontal gyrus and caudally into the postcentral gyrus.

accuracy in perceptual time judgment tasks (Keele et al., 1985). We therefore suggest that tapping variability represents a general temporal accuracy of neural activity, which is reflected in different timing tasks but which is also related to cognitive function. The main advantage of using a simple motor timing task, rather than a cognitive temporal judgment task, in the present study is that a relationship between intelligence and temporal variability can be demonstrated in a timing task that does not involve cognitive control or working memory.

Additional work is needed to understand the relationship between timing variability and cognitive performance. In general, one highly plausible possibility is clearly that dopaminergic mechanisms provide a link between the two phenomena (Lustig et al., 2005). Large bodies of literature show that dopaminergic neurotransmission is involved in both prefrontal functions related to cognition (Miller and Cohen, 2001; Nieoullon, 2002; Bäckman et al., 2006; Cropley et al., 2006) and timing (Rammsayer, 1997; Buhusi and Meck, 2005; Lewis and Miall, 2006; Meck, 2006). Administration of dopaminergic drugs has been shown to simultaneously affect timing and working memory (Buhusi and Meck, 2007).

However, the association of both timing variability and intel- 


$$
y=43
$$

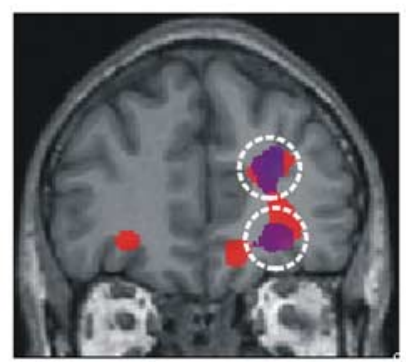

$$
x=26
$$

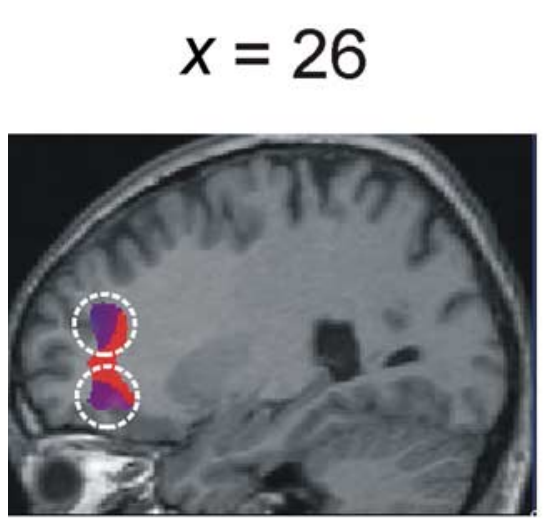

\section{Mid front $g$}
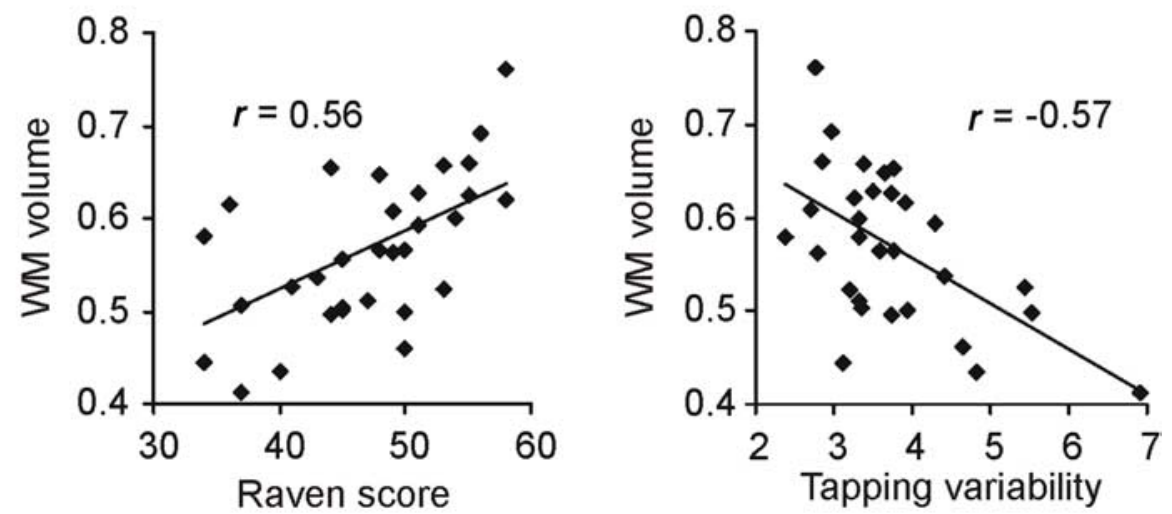
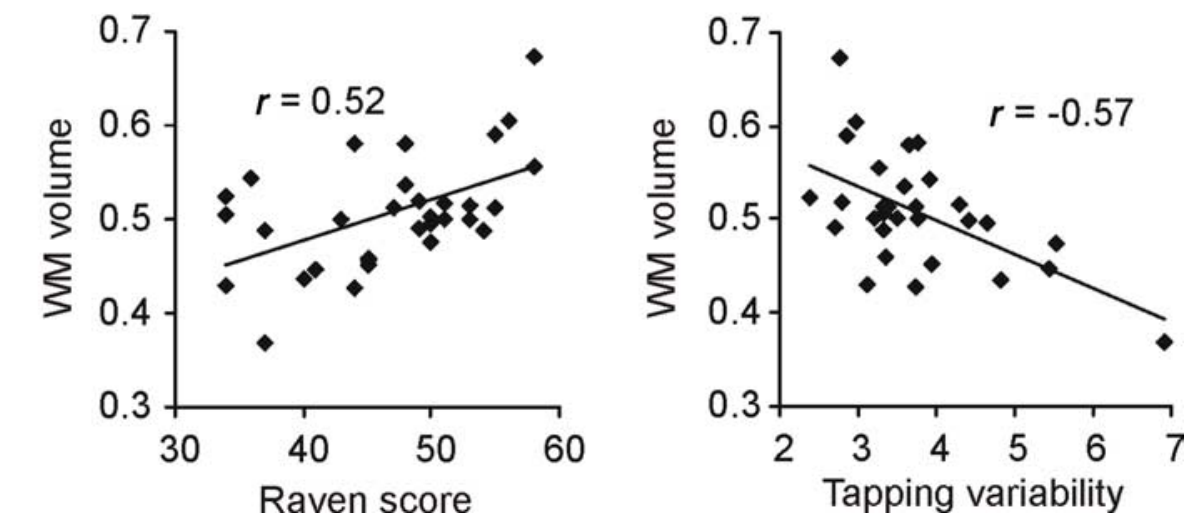

Figure 2. Right prefrontal white matter regions in which regional volume correlated negatively with tapping variability and positively with intelligence. Clusters are rendered on coronal and sagittal sections. Coordinates in standard space are given above each section. Red clusters show regions in which WMV correlated negatively with tapping variability ( $p<0.05$, corrected). Blue clusters show subregions, within these clusters, in which WMV also correlated positively with intelligence ( $p<0.05$, corrected). Scatter plots show correlations between regional volume (first eigenvariate of the cluster) and intelligence or temporal variability for the clusters in the right middle and inferior frontal gyri marked with dashed circles.

\begin{tabular}{|c|c|c|c|c|c|c|c|c|c|}
\hline \multirow[b]{2}{*}{ Brain region (nearest gray matter) } & \multirow[b]{2}{*}{ Side } & \multirow[b]{2}{*}{ Size $\left(\mathrm{mm}^{3}\right)$} & \multirow[b]{2}{*}{$t$ value } & \multirow[b]{2}{*}{$x$} & \multirow[b]{2}{*}{$y$} & \multirow[b]{2}{*}{$z$} & \multicolumn{3}{|c|}{ Explained portion of variance in intelligence } \\
\hline & & & & & & & WMV (unique) & CV (unique) & Commonality \\
\hline Frontal pole & $\mathrm{R}$ & 274 & 3.44 & 16 & 55 & -12 & 0.07 & 0.00 & 0.15 \\
\hline Middle frontal g & $\mathrm{R}$ & 1041 & 4.48 & 26 & 46 & 19 & 0.15 & 0.00 & 0.15 \\
\hline Inf frontal g/orbitofrontal cortex & $\mathrm{R}$ & 1113 & 3.76 & 26 & 39 & -12 & 0.13 & 0.00 & 0.15 \\
\hline Middle orbitofrontal g & $\mathrm{L}$ & 19 & 3.08 & -24 & 31 & -16 & 0.09 & 0.00 & 0.15 \\
\hline
\end{tabular}

Table 3. White matter regions in which regional volume correlated positively with intelligence and negatively with tapping variability

Significantly correlated $(p<0.05)$ regions with an extent of more than five voxels are reported. All peaks remained significant when controlling for age. The coordinates in MNI space and $t$ value of the peak voxel of each cluster are given. The three rightmost columns give, for each cluster, the proportion of total variance in Raven scores explained by cluster WMV and CV scores uniquely, as well as by common effects of these two variables. $g$, Gyrus; Inf, inferior; L, left; R, right.

ligence with increased prefrontal white matter found in the present study also suggests an additional possibility. A larger prefrontal white matter volume presumably reflects a larger number of corticocortical connections. Glutamatergic corticocortical connections have been shown to be of importance for synchronization of cortical neural activity (Traub et al., 2004). Furthermore, a large literature demonstrates that coordination of neuronal activity in the millisecond range, within and between brain regions, is essential for a broad range of cognitive functions, such as working memory, perceptual binding and awareness, and attention (Singer, 1999; Fries, 2005; Uhlhaas and Singer, 2006). We therefore propose that increased prefrontal connectivity may lead to more temporally stable neural activity, which is reflected in automatic timing tasks, but more importantly gives a generally increased capacity to form temporally well coordinated discharges in prefrontal neuronal networks. This in turn could affect intelligence through to its effects on the performance of cognitive operations that are dependent on neural synchrony in the millisecond range.

\section{References}

Bäckman L, Nyberg L, Lindenberger U, Li SC, Farde L (2006) The correlative triad among aging, dopamine, and cognition: current status and future prospects. Neurosci Biobehav Rev 30:791-807.

Baumeister AA (1998) Intelligence and the "personal equation." Intelligence 26:255-265. 
Buhusi CV, Meck WH (2005) What makes us tick? Functional and neural mechanisms of interval timing. Nat Rev Neurosci 6:755-765.

Buhusi CV, Meck WH (2007) Effect of clozapine on interval timing and working memory for time in the peak-interval procedure with gaps. Behav Processes 74:159-167.

Colom R, Jung RE, Haier RJ (2006) Distributed brain sites for the g-factor of intelligence. NeuroImage 31:1359-1365.

Cropley VL, Fujita M, Innis RB, Nathan PJ (2006) Molecular imaging of the dopaminergic system and its association with human cognitive function. Biol Psychiatry 59:898-907.

Cuadra MB, Cammoun L, Butz T, Cuisenaire O, Thiran JP (2005) Comparison and validation of tissue modelization and statistical classification methods in T1-weighted MR brain images. IEEE Trans Med Imaging 24:1548-1565.

Deary IJ (2000) Looking down on human intelligence: from psychometrics to the brain. Oxford: Oxford UP.

Deary IJ (2001) Human intelligence differences: towards a combined experimental-differential approach. Trends Cogn Sci 5:164-170.

Duncan J, Seitz RJ, Kolodny R, Bor D, Herzog H, Ahmen A, Newell FN, Emslie H (2000) A neural basis for general intelligence. Science 289:399-401.

Frangou S, Chitins X, Williams SC (2004) Mapping IQ and gray matter density in healthy young people. NeuroImage 23:800-805.

Fries P (2005) A mechanism for cognitive dynamics: neuronal communication through neuronal coherence. Trends Cogn Sci 474-480.

Geake JG, Hansen PC (2005) Neural correlates of intelligence as revealed by fMRI of fluid analogies. NeuroImage 26:555-564.

Genovese CR, Lazar NA, Nichols T (2002) Thresholding of statistical maps in functional neuroimaging using the false discovery rate. NeuroImage 15:870-878.

Goel V, Dolan RJ (2001) Functional neuroanatomy of three-term relational reasoning. Neuropsychologia 39:901-909.

Goel V, Gold B, Kapur S, Houle S (1997) The seats of reason? An imaging study of deductive and inductive reasoning. NeuroReport 8:1305-1310.

Good CD, Johnsrude IS, Ashburner J, Henson RNA, Friston KJ, Frackowiak RSJ (2001) A voxel-based morphometric study of ageing in 465 normal adult human brains. NeuroImage 14:21-36.

Gustafsson J-E (1984) A unifying model for the structure of intellectual abilities. Intelligence 8:179-203.

Haier RJ, Siegel BV, Nuechterlein KH, Hazlett E, Wu JC, Paek J, Browning HL, Buchsbaum MS (1988) Cortical glucose metabolic rate correlates of abstract reasoning and attention studied with positron emission tomography. Intelligence 12:199-217.

Haier RJ, Jung RE, Yeo RA, Head K, Alkire MT (2004) Structural brain variation and general intelligence. NeuroImage 23:425-433.

Haier RJ, Jung RE, Yeo RA, Head K, Alkire MT (2005) The neuroanatomy of general intelligence: sex matters. NeuroImage 25:320-327.

Helmbold N, Troche S, Rammsayer T (2007) Processing of temporal and nontemporal information as predictors of psychometric intelligence: a structural-equation-modeling approach. J Pers 75:985-1006.

Jensen AR (1992) The importance of intraindividual variation in reactiontime. Personal Individ Diff 13:869-881.

Jensen AR (2006) Clocking the mind: mental chronometry and individual differences. Oxford: Elsevier.

Jung RE, Haier RJ (2007) The parieto-frontal integration theory (P-FIT) of intelligence: converging neuroimaging evidence. Behav Brain Sci 30:135-154.

Keele SW, Pokorny RA, Corcos DM, Ivry R (1985) Do perception and motor production share common timing mechanisms: a correlational analysis. Acta Psychol 60:173-191.

Kroger JK, Sabb FW, Fales CL, Bookheimer SY, Cohen MS, Holyoak KJ (2002) Recruitment of anterior dorsolateral prefrontal cortex in human reasoning: a parametric study of relational complexity. Cereb Cortex 12:477-485.

Kyllonen PC, Christal RE (1990) Reasoning ability is (little more than) working memory capacity? Intelligence 14:389-433.
Lewis PA, Miall RC (2003) Distinct systems for automatic and cognitively controlled time measurement: evidence from neuroimaging. Curr Opin Neurobiol 13:250-255.

Lewis PA, Miall RC (2006) Remembering the time: a continuous clock. Trends Cogn Sci 10:401-406.

Lustig C, Matell MS, Meck WH (2005) Not “just" a coincidence: frontalstriatal interactions in working memory and interval timing. Memory 13:441-448.

Madison G (2001) Variability in isochronous tapping: higher-order dependencies as a function of inter tap interval. J Exp Psychol Hum Percept Perf 27:411-422.

Madison G, Merker B (2004) Human sensorimotor tracking of continuous subliminal deviations from isochrony. Neurosci Lett 370:69-73.

McDaniel MA (2005) Big-brained people are smarter: a meta-analysis of the relationship between in vivo brain volume and intelligence. Intelligence 33:337-346.

Meck WH (2006) Neuroanatomical localization of an internal clock: a functional link between mesolimbic, nigrostriatal, and mesocortical dopaminergic systems. Brain Res 1109:93-107.

Miller EK, Cohen JD (2001) An integrative theory of prefrontal cortex function. Annu Rev Neurosci 24:167-202.

Mühlau M, Rauschecker JP, Oestreicher E, Gaser C, Rottinger M, Wohlschlager AM, Simon F, Etgen T, Conrad B, Sander D (2006) Structural brain changes in tinnitus. Cereb Cortex 16:1283-1288.

Nieoullon A (2002) Dopamine and the regulation of cognition and attention. Prog Neurobiol 67:53-83.

Oldfield RC (1971) The assessment and analysis of handedness: the Edinburgh inventory. Neuropsychologia 9:97-113.

Prabhakaran V, Smith JA, Desmond JE, Glover GH, Gabrieli JD (1997) Neural substrates of fluid reasoning: an fMRI study of neocortical activation during performance of the Raven's Progressive Matrices Test. Cognit Psychol 33:43-63.

Rammsayer TH (1997) Are there dissociable roles of the mesostriatal and mesolimbocortical dopamine systems on temporal information processing in humans? Neuropsychobiology 35:36-45.

Rammsayer TH, Brandler S (2007) Performance on temporal information processing as an index of general intelligence. Intelligence 35:123-139.

Reiss AL, Abrams MT, Singer HS, Ross JL, Denckla MB (1996) Brain development, gender and IQ in children. A volumetric imaging study. Brain 119:1763-1774.

Repp BH (2006) Does an auditory distractor sequence affect self-paced tapping? Acta Psychol 121:81-107.

Rushton JP, Ankney CD (2007) The evolution of brain size and intelligence. In: Evolutionary cognitive neuroscience (Platek SM, Keenan JP, Shackelford TK, eds), pp 121-161. Cambridge, MA: MIT.

Seibold DR, McPhee RD (1979) Commonality analysis: a method for decomposing explained variance in regression analyses. Hum Commun Res 5:355-365.

Shaw P, Greenstein D, Lerch J, Clasen L, Lenroot R, Gogtay N, Evans A, Rapoport J, Giedd JN (2006) Intellectual ability and cortical development in children and adolescents. Nature 440:676-679.

Singer W (1999) Neuronal synchrony: a versatile code of the definition of relations? Neuron 24:49-65.

Styles I, Raven M, Raven JC (1998) Raven's progressive matrices. SPM plus sets A-E. Oxford: Oxford Psychologists.

Traub RD, Bibbig A, LeBeau FE, Buhl EH, Whittington MA (2004) Cellular mechanisms of neuronal population oscillations in the hippocampus in vitro. Annu Rev Neurosci 27:247-278.

Uhlhaas PJ, Singer W (2006) Neural synchrony in brain disorders: relevance for cognitive dysfunctions and pathophysiology. Neuron 52:155-168.

Vorberg D, Wing A (1996) Modeling variability and dependence in timing. In: Handbook of perception and action, pp 181-262. New York: Academic.

Walhovd KB, Fjell AM (2007) White matter volume predicts reaction time instability. Neuropsychologia 45:2277-2284. 